\title{
Flow Cytometric Analysis of T, B, and NK Cells Antigens in Patients with Mycosis Fungoides
}

\author{
Serkan Yazıcı, ${ }^{1}$ Emel Bülbül Başkan, ${ }^{1}$ Ferah Budak, ${ }^{2}$ Barbaros Oral, ${ }^{2}$ \\ Şaduman Balaban Adim, ${ }^{3}$ Zübeyde Ceylan Kalin, ${ }^{1}$ Güven Özkaya, ${ }^{4}$ Kenan Aydoğan, \\ Hayriye Saricaoğlu, ${ }^{1}$ and Şükran Tunali ${ }^{1}$ \\ ${ }^{1}$ Uludag University School of Medicine, Department of Dermatology and Venereology, Bursa, Turkey \\ ${ }^{2}$ Uludag University School of Medicine, Department of Immunology, Bursa, Turkey \\ ${ }^{3}$ Uludag University School of Medicine, Department of Pathology, Bursa, Turkey \\ ${ }^{4}$ Uludag University School of Medicine, Department of Biostatistics, Bursa, Turkey \\ Correspondence should be addressed to Serkan Yazıc1; serkanyazici@uludag.edu.tr
}

Received 22 June 2015; Revised 4 November 2015; Accepted 24 November 2015

Academic Editor: Takami Sato

Copyright (C) 2015 Serkan Yazıcı et al. This is an open access article distributed under the Creative Commons Attribution License, which permits unrestricted use, distribution, and reproduction in any medium, provided the original work is properly cited.

\begin{abstract}
We retrospectively analyzed the clinicopathological correlation and prognostic value of cell surface antigens expressed by peripheral blood mononuclear cells in patients with mycosis fungoides (MF). 121 consecutive MF patients were included in this study. All patients had peripheral blood flow cytometry as part of their first visit. TNMB and histopathological staging of the cases were retrospectively performed in accordance with International Society for Cutaneous Lymphomas/European Organization of Research and Treatment of Cancer (ISCL/EORTC) criteria at the time of flow cytometry sampling. To determine prognostic value of cell surface antigens, cases were divided into two groups as stable and progressive disease. 17 flow cytometric analyses of 17 parapsoriasis (PP) and 11 analyses of 11 benign erythrodermic patients were included as control groups. Fluorescent labeled monoclonal antibodies were used to detect cell surface antigens: $\mathrm{T}$ cells $\left(\mathrm{CD}^{+}, \mathrm{CD}^{+}, \mathrm{CD} 8^{+}, \mathrm{TCR} \alpha \beta^{+}, \mathrm{TCR} \gamma \delta^{+}, \mathrm{CD} 7^{+}, \mathrm{CD} 4^{+} \mathrm{CD} 7^{+}, \mathrm{CD}^{+} \mathrm{CD}^{-}\right.$, and $\left.\mathrm{CD}_{1}{ }^{+}\right), \mathrm{B}$ cells $\left(\mathrm{HLA}-\mathrm{DR}{ }^{+}, \mathrm{CD} 19^{+}\right.$, and HLA-DR $\left.{ }^{+} \mathrm{CD} 19^{+}\right)$, NKT cells $\left(\mathrm{CD} 3^{+} \mathrm{CD} 16^{+} \mathrm{CD} 56^{+}\right)$, and NK cells $\left(\mathrm{CD} 3^{-} \mathrm{CD} 16^{+} \mathrm{CD}^{+} 6^{+}\right)$. The mean value of all cell surface antigens was not statistically significant between parapsoriasis and MF groups. Along with an increase in cases of MF stage statistically significant difference was found between the mean values of cell surface antigens. Flow cytometric analysis of peripheral blood cell surface antigens in patients with mycosis fungoides may contribute to predicting disease stage and progression.
\end{abstract}

\section{Introduction}

Mycosis fungoides is the most common variant of primary cutaneous $\mathrm{T}$ cell lymphomas. Most of MF patients first present with long-standing reactive inflammatory dermatoses such as PP en plaque so-called premycotic eruptions [1]. Flow cytometric analysis of the cell surface antigens, expressed by peripheral blood cells, is widely used in the diagnosis and also management of hematologic malignancies; however, it has not been used routinely in the evaluation of MF patients. The diagnosis of MF is still challenging with current histopathological and molecular techniques especially in early stages [2]. Neoplastic T cells frequently have an altered level of expression of various surface $\mathrm{T}$ cell markers compared with normal $\mathrm{T}$ cells. These differences are sufficient to distinguish normal $\mathrm{T}$ cells from neoplastic $\mathrm{T}$ cells in the same population [3]. Peripheral blood flow cytometry can detect aberrant $\mathrm{T}$ cell populations even when there is no lymphocytosis or elevated total white blood cell count. Detection of T cell abnormalities by flow cytometry is increasing in use as an effective and a sensitive method in MF patients [4]. The importance of peripheral blood flow cytometry studies is highlighted by the ISCL/EORTC recommendations for diagnosis that include one or more of the following phenotypical abnormalities demonstrated by flow cytometry including peripheral blood $\mathrm{CD} 4^{+} / \mathrm{CD}^{+}$ rate being above 10, abnormal expression of pan- $\mathrm{T}$ cell markers including $\mathrm{CD} 2^{+}, \mathrm{CD}^{+}, \mathrm{CD} 4^{+}$antigens, and $\mathrm{CD} 7$ 
TABle 1: Demographic features of the groups included in the study.

\begin{tabular}{lccr}
\hline & Mycosis fungoides & Parapsoriasis & Benign erythroderma \\
\hline$n /$ flow analysis & $121 / 121$ & $17 / 17$ & $11 / 11$ \\
Gender & & & \\
$\quad$ \&/ð & $51 / 70$ & $11 / 6$ & $6 / 5$ \\
Flow age (y) & & & $62.23 \pm 15.78$ \\
$\quad$ Mean \pm SD & $54.42 \pm 13.81$ & $46.99 \pm 12.05$ & $68.50 ; 36-83$ \\
$\quad$ Median; min.-max. & $54.0 ; 18-90$ & $46.00 \pm 17-62$ & - \\
Duration of disease (y) & & & $1 ; 1-20$ \\
$\quad$ Mean \pm SD & $7.14 \pm 5.60$ & $2.94 \pm 2.46$ & $2.0 \pm 1-9$ \\
$\quad$ Median; min.-max. & $5.0 ; 1-35$ & & \\
\hline
\end{tabular}

$\left(\mathrm{CD} 4^{+} \mathrm{CD}^{-} \geq 40 \%\right)$ antigen loss of $\mathrm{T}$ cells [5]. Aberrant expression of $\mathrm{T}$ cells, and also $\mathrm{B}$ and NK cells markers, may be expressed in patients with $\mathrm{MF}[6,7]$.

The aim of this study was to evaluate the prognostic value of cell surface antigens expressed by T, B, and NK cells and correlated the results with the clinicopathological ISCL MF diagnostic score, retrospectively by 4 -color flow cytometry.

\section{Patients and Methods}

2.1. Patients. A total of 121 flow cytometric analyses of $121 \mathrm{MF}$ patients, followed up in our clinic between January 2000 and August 2013, were enrolled in the study. All patients had peripheral blood flow cytometry as part of their first visit. TNMB and histopathological staging of the cases were performed in accordance with International Society for Cutaneous Lymphomas/European Organization of Research and Treatment of Cancer (ISCL/EORTC) criteria at the time of flow cytometry sampling retrospectively. 17 flow cytometric analyses of $17 \mathrm{PP}$ and 11 analyses of 11 benign erythrodermic patients were included as control groups (Table 1). By the date of each flow sytometric analysis, TNMB and histopathological staging of cases were performed to all patients according to the Bunn-Lambert staging system and ISCL/EORTC criteria were retrieved from the medical records [5]. To determine prognostic value of flow cytometric analysis, according to the course of the follow-up process of the patients, cases were divided into two groups stable and progressive disease as recommended. Stable disease is described as $<25 \%$ increase to $<50 \%$ clearance in skin disease from baseline without new tumors $\left(\mathrm{T}_{3}\right)$ in patients with $\mathrm{T}_{1}$, $\mathrm{T}_{2}$, or $\mathrm{T}_{4}$ only skin disease. Progressive disease is described as $\geq 25 \%$ increase in skin disease from baseline or new tumors $\left(\mathrm{T}_{3}\right)$ in patients with $\mathrm{T}_{1}, \mathrm{~T}_{2}$, or $\mathrm{T}_{4}$ only skin disease or loss of response: in those with complete or partial response, increase of skin score of greater than the sum of plus $50 \%$ baseline score [8]. The study was approved by the local ethical committee and conducted according to the principles of the Declaration of Helsinki.

2.2. Flow Cytometric Analysis. Peripheral blood specimens were collected in EDTA tubes. Blood samples were transported to the flow cytometry laboratory where the specimens were processed and analyzed within 24 hours of receipt. After incubation of whole peripheral blood with monoclonal antibodies for 15 minutes at room temperature in the dark, erythrocytes were lysed with NH4CL for $10 \mathrm{~min}$, followed by two washing steps using phosphate buffered saline solution. The cells were then resuspended and fixed with $1 \%$ paraformaldehyde.

Four-color flow cytometry was performed using FACSCanto (BD Biosciences, USA). Four-color direct immunofluorescent staining was performed as described by the manufacturer, using a 7-tube panel $(70 \times 15 \mathrm{~mm})$. $100 \mu \mathrm{L}\left(10^{5}\right.$ cells) blood samples, containing EDTA, were transferred to tubes and the tubes were placed in the Lyse Wash Assistant (LWA) (BD Biosciences, USA). Fluorescent labeled monoclonal antibodies were used to detect cell surface antigens: $T$ cells $\left(\mathrm{CD}^{+}, \mathrm{CD}^{+}, \mathrm{CD} 8^{+}, \mathrm{TCR} \alpha \beta^{+}\right.$, $\mathrm{TCR} \gamma \delta^{+}, \mathrm{CD}^{+}, \mathrm{CD} 4^{+} \mathrm{CD}^{+}, \mathrm{CD}^{+}{ }^{+} \mathrm{CD} 7^{-}$, and $\left.\mathrm{CD}^{+} 1^{+}\right), B$ cells (HLA-DR ${ }^{+}, \mathrm{CD}_{1} 9^{+}$, and $\left.\mathrm{HLA}-\mathrm{DR}^{+} \mathrm{CD} 19^{+}\right), \mathrm{NKT}$ cells $\left(\mathrm{CD}^{+} \mathrm{CD}^{+} 6^{+} \mathrm{CD}^{+} 6^{+}\right)$, and $\mathrm{NK}$ cells $\left(\mathrm{CD} 3^{-} \mathrm{CD} 16^{+} \mathrm{CD} 56^{+}\right)$. Fluorescein isothiocyanate (FITC), phycoerythrin (PE), Allophycocyanin (APC), and Peridinin-ChlorophyllProtein-Complex (PerCP) labeled fluorescent monoclonal antibodies (Beckman Coulter, France) were used. Cells were collected and analyzed on a FACSCanto (BD Biosciences, USA) using FACSDiva (BD Biosciences, USA). The tubes were aspirated until dry to maximize cell yield. Lymphocytes were identified in the standard manner based on CD45 expression and side angle light scatter.

2.3. Statistical Analysis. SPSS for Windows Version 20.0 (SPSS Inc, Chicago, Illinois, USA) software package program was used for the statistical analysis. Variables were represented with mean, standard deviation, median, and maximum-minimum values. Categorical values of the variables given to cross-tables and differences between the two groups were analysed with Pearson's chi-square and Fisher's exact test. Median and mean values of binary groups compared with using Kruskal-Wallis and Mann-Whitney tests, and for more than two groups ANOVA test was used. ROC curve analysis was performed to determine the cut-off values between progressive and stable disease groups. $P<0.05$ was considered to be significant.

\section{Results}

A total of $121 \mathrm{MF}$ patients $(70 \mathrm{M}, 51 \mathrm{~F})$ were included in the study. The age of MF patients during sampling ranged 
TABLE 2: Clinical and demographic features of MF patients and histopathological and TNM staging of cases according to the BunnLambert staging system and ISCL/EORTC criteria.

\begin{tabular}{|c|c|}
\hline$n$ & 121 \\
\hline \multicolumn{2}{|l|}{ Gender } \\
\hline \$/రి & $51 / 70$ \\
\hline \multicolumn{2}{|l|}{ Age (y) } \\
\hline Mean \pm SD & $54.42 \pm 13.81$ \\
\hline Median; min.-max. & $54.0 ; 18-90$ \\
\hline \multicolumn{2}{|l|}{ Duration of disease (y) } \\
\hline Mean \pm SD & $7.14 \pm 5.60$ \\
\hline Median; min.-max. & $5.0 ; 1-35$ \\
\hline \multicolumn{2}{|l|}{ Follow-up period $(y)$} \\
\hline Mean \pm SD & $4.28 \pm 2.96$ \\
\hline Median; min.-max. & $4 ; 1-14$ \\
\hline \multicolumn{2}{|l|}{ Coursing } \\
\hline Stable & 105 \\
\hline Progressive & 16 \\
\hline Eksitus & 5 \\
\hline \multicolumn{2}{|l|}{ Histopathological stage } \\
\hline Patch & 75 \\
\hline Plaque & 37 \\
\hline Tumor & 9 \\
\hline \multicolumn{2}{|l|}{ Clinical $(\mathrm{T})$ stage } \\
\hline $\mathrm{T}_{1}$ & 33 \\
\hline $\mathrm{T}_{2}$ & 68 \\
\hline $\mathrm{T}_{3}$ & 7 \\
\hline $\mathrm{T}_{4}$ & 13 \\
\hline \multicolumn{2}{|c|}{ Lymph node $(\mathrm{N})$ involvement } \\
\hline None & 109 \\
\hline Positive & 12 \\
\hline \multicolumn{2}{|l|}{ TNM staging } \\
\hline \multicolumn{2}{|l|}{ Early (IA -IIA) } \\
\hline IA & 33 \\
\hline IB & 67 \\
\hline IIA & 1 \\
\hline \multicolumn{2}{|l|}{ Late (IIB-IV) } \\
\hline IIB & 6 \\
\hline III & 10 \\
\hline IV & 4 \\
\hline
\end{tabular}

from 18 to 89 years (mean $\pm \mathrm{SD} ; 54.42 \pm 13.81$ ). The age of 17 parapsoriasis patients $(6 \mathrm{M}, 11 \mathrm{~F})$ ranged from 17 to 62 (mean $\pm \mathrm{SD} ; 46.99 \pm 12.05$ ). The age of benign erythroderma patients ranged from 36 to 83 . MF patients were followed up for an average of $4.28 \pm 2.96$ years, 105 cases were accepted as stable, and 16 cases were progressive. Clinical and demographic features of MF patients and histopathological and TNM staging of cases according to the Bunn-Lambert staging system and ISCL/EORTC criteria were summarized in Table 2.

The mean value of all cell surface antigens was not statistically significant between parapsoriasis and MF groups. This finding may show that flow cytometry is not enough alone to differentiate mycosis fungoides from benign dermatoses and parapsoriasis. $\mathrm{CD}^{+} \mathrm{CD}^{-} 6^{+} \mathrm{CD} 56^{+}$cells may differentiate benign erythrodermic and MF patients assessed with twosided $t$-test $(P=0.027)$ (Table 3$)$. According to histopathological and clinical stage in cases of mycosis fungoides, statistically significant differences were found between the mean values of all cell surface antigens (Tables 4 and 5). While increasing stage of disease the number of $\mathrm{CD}^{+}$, $\mathrm{CD} 4^{+}, \mathrm{TCR} \alpha \beta^{+}, \mathrm{CD} 4^{+} \mathrm{CD}^{+}, \mathrm{CD}^{+} \mathrm{CD}^{-}$, and $\mathrm{CD}^{+} 1^{+}$cells and $\mathrm{CD} 4^{+} / \mathrm{CD}^{+}$cell percentage were significantly increased and the number of $\mathrm{CD}^{+}, \mathrm{CD}^{+}, \mathrm{HLA}^{-\mathrm{DR}}{ }^{+}, \mathrm{CD} 19^{+}, \mathrm{HLA}-$ $\mathrm{DR}^{+}, \mathrm{CD} 19^{+}, \mathrm{CD}^{-} \mathrm{CD} 16^{+}$, and $\mathrm{CD} 56^{+}$cells decreased significantly (Table 5). These findings may be useful in identifying advanced stage cases in patients with MF.

When MF patients were examined in two groups as stable and progressive, all cell surface antigens except $\mathrm{CD} 4^{+} \mathrm{CD}^{-}$ cells may play a role in determining the progression. According to ROC curve analysis results, flow cytometric results suggestive of disease progression were as follows: mean $\mathrm{CD}^{+}$ cell percentage $>79.1$; mean $\mathrm{CD}^{+}$cell percentage $>49.2$; mean $\mathrm{CD}^{+}$cell percentage $\leq 5.2 ; \mathrm{CD}^{+} / \mathrm{CD} 8^{+}$percentage $>2.4$; mean $\mathrm{TCR} \alpha \beta^{+}$cell percentage $>73.1 ; \mathrm{TCR} \gamma \delta^{+}$cell percentage $\leq 2.4 ; \mathrm{CD}^{+}$cell percentage $\leq 48 ; \mathrm{CD} 4^{+} \mathrm{CD} 7^{+}$cell percentage $>47.8 ; \mathrm{CD}^{+} \mathrm{CD}^{-}$cell percentage $>9.4 ; \mathrm{CD}^{+} 1^{+}$ cell percentage $>3.4 ; \mathrm{HLA}_{-} \mathrm{DR}^{+}$cell percentage $\leq 23.1 \mathrm{CD}^{+}{ }^{+}$ cell percentage $\leq 6$; $\mathrm{HLA}-\mathrm{DR}^{+} \mathrm{CD} 19^{+}$cell percentage $\leq 5$; $\mathrm{CD}^{+}{ }^{+} \mathrm{CD} 16^{+} \mathrm{CD} 6^{+}$cell percentage $\leq 1$; $\mathrm{CD} 3^{-} \mathrm{CD} 16^{+} \mathrm{CD} 56^{+}$ cell percentage $\leq 9.9$ in peripheral blood at any time (Table 6).

\section{Discussion}

The diagnosis of MF remains a challenging area in dermatopathology and conclusive diagnosis is often difficult, necessitating the terms "diagnostic of," "consistent with," and "suggestive of" to describe the disease and histopathologic finding needs to be confirmed with the immunohistochemical studies with some limitations in between [9]. The cluster of differentiation (termed as Cluster of Designation or Classification Determinant) (CD) is a protocol used for the identification and investigation of cell surface molecules for immunophenotyping of cells. The use of combining markers has allowed more specific definitions for cell types within the immune system than one molecule $[10,11]$. Previous studies using flow cytometry to analyze skin biopsy specimens for the diagnosis of CTCL and MF have been performed and reported that flow cytometry is a highly sensitive and specific diagnostic test for MF $[4,12,13]$. In addition to skin biopsy specimens, flow cytometric analysis has proven to be an efficient and sensitive method to detect and enumerate MF/SS cells in the peripheral blood $[3,14,15]$. Depending on the degree of differentiation of neoplastic cells one or more altered expressions of $\mathrm{T}$ cell related antigens including $\mathrm{CD} 2^{+}$, $\mathrm{CD}^{+}, \mathrm{CD}^{+}, \mathrm{CD}^{+}$, or $\mathrm{CD} 26^{+}$, were identified in $92 \%$ of cases as positive for malignant $\mathrm{T}$ cell clones, with loss of $\mathrm{CD}^{2} 6^{+}$being the most common $(70 \%)$, and $\mathrm{CD}^{+} 6^{+}$cases have been reported $[16,17]$. However, increased $\mathrm{CD}^{2} 6^{+}$cells, or $\mathrm{CD}^{+}$cells, and even altered expression levels of other more specific $\mathrm{T}$ cell markers, such as $\mathrm{CD}^{+}$and $\mathrm{CD} 4^{+}$, were also observed in nonneoplastic samples. That loss or dim 
TABLE 3: The mean value of all cell surface antigens between groups.

\begin{tabular}{|c|c|c|c|c|}
\hline CD molecule & $\begin{array}{c}\text { Mycosis fungoides } \\
n: 121 \\
\end{array}$ & $\begin{array}{c}\text { Parapsoriasis } \\
n: 17\end{array}$ & $\begin{array}{c}\text { Benign erythroderma } \\
n: 11\end{array}$ & \\
\hline $\mathrm{CD}^{+}$ & & & & 0.917 \\
\hline Mean \pm SD & $73.66 \pm 9.62$ & $71.12 \pm 3.33$ & $62.97 \pm 23.26$ & \\
\hline Median; min.-max. & $73.60 ; 48.70-98.70$ & $70.90 ; 67.70-74.70$ & $58.70 ; 43.60-90.90$ & \\
\hline $\mathrm{CD}_{4}^{+}$ & & & & 0.754 \\
\hline Mean \pm SD & $47.54 \pm 13.26$ & $43.0 \pm 8.81$ & $39.62 \pm 27.09$ & \\
\hline Median; min.-max. & $45.3 \pm 15.30-97.50$ & $48.70 ; 31.20-50.40$ & $32.05 ; 15,80-78.60$ & \\
\hline $\mathrm{CD}^{+}$ & & & & 0.954 \\
\hline Mean \pm SD & $23.49 \pm 10.62$ & $25.96 \pm 4.86$ & $20.95 \pm 9.86$ & \\
\hline Median; min.-max. & $22.0 ; 0.50-73.30$ & $24.9 ; 21.40-32.60$ & $20.3 ; 10.60-32.60$ & \\
\hline $\mathrm{CD}^{+} / \mathrm{CD}^{+}$ & & & & 0.836 \\
\hline Mean \pm SD & $5.61 \pm 18.69$ & $1.74 \pm 0.6$ & $2.40 \pm 2.09$ & \\
\hline Median; min.-max. & $2.0 ; 0.2-183.2$ & $2.0 ; 0.90-2.30$ & $1.95 ; 0.60-5.10$ & \\
\hline $\operatorname{TCR} \alpha \beta^{+}$ & & & & 0.944 \\
\hline Mean \pm SD & $68.96 \pm 9.66$ & $67.88 \pm 4.0$ & $58.80 \pm 20.71$ & \\
\hline Median; min.-max. & $69.10 ; 45.80-98.0$ & $69.60 ; 63.10-72.50$ & $53.35 ; 42.0-86.50$ & \\
\hline $\mathrm{TCR} \gamma \delta^{+}$ & & & & 0.056 \\
\hline Mean \pm SD & $4.09 \pm 2.91$ & $3.10 \pm 2.10$ & $3.35 \pm 4.43$ & \\
\hline Median; min.-max. & $3.20 ; 0.10-13.60$ & $4.30 ; 0.80-4.80$ & $1.20 ; 1.0-10.0$ & \\
\hline $\mathrm{CD}^{+}$ & & & & 0.585 \\
\hline Mean \pm SD & $74.22 \pm 12.60$ & $80.0 \pm 3.77$ & $77.4 \pm 4.35$ & \\
\hline Median; min.-max. & $76.60 ; 2.80-98.0$ & $79.2 ; 74.60-84.0$ & $79.05 ; 71.10-80.40$ & \\
\hline $\mathrm{CD} 4^{+} \mathrm{CD}^{+}$ & & & & 0.478 \\
\hline Mean \pm SD & $38.98 \pm 11.50$ & $40.68 \pm 7.40$ & $37.22 \pm 17.86$ & \\
\hline Median; min.-max. & $38.60 ; 9.60-96.50$ & $44.80 ; 31.10-46.80$ & $31.75 ; 22.30-63.10$ & \\
\hline $\mathrm{CD} 4^{+} \mathrm{CD}^{-}$ & & & & 0.193 \\
\hline Mean \pm SD & $9.62 \pm 11.53$ & $4.52 \pm 1.43$ & $4.95 \pm 3.87$ & \\
\hline Median; min.-max. & $5.60 ; 0.50-88.90$ & $4.90 ; 2.20-6.10$ & $3.40 ; 2.30-10.70$ & \\
\hline $\mathrm{CD}_{71}^{+}$ & & & & 0.091 \\
\hline Mean \pm SD & $5.70 \pm 9.98$ & $1.80 \pm 0.71$ & $15.65 \pm 23.69$ & \\
\hline Median; min.-max. & $3.0 ; 0.1-71.0$ & $1.90 ; 0.8-2.80$ & $4.95 ; 1.6-51.1$ & \\
\hline \multicolumn{5}{|c|}{ B cell surface antigens } \\
\hline HLA-DR ${ }^{+}$ & & & & 0.368 \\
\hline Mean \pm SD & $30.47 \pm 10.05$ & $28.06 \pm 5.81$ & $37.22 \pm 16.46$ & \\
\hline Median; min.-max. & $29.2 ; 2.20-60.20$ & $26.4 ; 20.6-36.0$ & $33.6 ; 21.80-59.90$ & \\
\hline $\mathrm{CD} 19^{+}$ & & & & 0.468 \\
\hline Mean \pm SD & $11.66 \pm 4.81$ & $12.74 \pm 4.77$ & $9.40 \pm 3.84$ & \\
\hline Median; min.-max. & $11.70 ; 1.0-24.80$ & $10.90 ; 8.90-20.80$ & $9.95 ; 4.90-12.80$ & \\
\hline 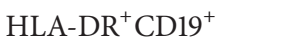 & & & & 0.559 \\
\hline Mean \pm SD & $10.80 \pm 4.80$ & $12.22 \pm 4.70$ & $8.20 \pm 3.34$ & \\
\hline Median; min.-max. & $10.70 ; 0.30-24.70$ & $10.40 ; 8.70-20.10$ & $50 ; 4.60-11.20$ & \\
\hline \multicolumn{5}{|c|}{ NKT cells surface antigens } \\
\hline $\mathrm{CD}^{+}{ }^{+} \mathrm{CD} 16^{+} \mathrm{CD} 56^{+}$ & & & & 0.027 \\
\hline Mean \pm SD & $3.31 \pm 3.26$ & $1.54 \pm 0.78$ & $1.92 \pm 1.30$ & \\
\hline Median; min.-max. & $2.20 ; 0.20-20.50$ & $1.20 ; 0.90-2.80$ & $1.95 ; 0.70-3.10$ & \\
\hline \multicolumn{5}{|c|}{ NK cells surface antigens } \\
\hline $\mathrm{CD}^{-} \mathrm{CD}^{-} 6^{+} \mathrm{CD} 56^{+}$ & & & & 0.523 \\
\hline Mean \pm SD & $12.92 \pm 8.06$ & $13.56 \pm 5.28$ & $16.87 \pm 1.83$ & \\
\hline Median; min.-max. & $12.80 ; 0.10-39.80$ & $13.60 ; 7.30-21.50$ & $17.55 ; 2.20-30.20$ & \\
\hline
\end{tabular}


TABLE 4: The mean value of all cell surface antigens according to histopathological stage.

\begin{tabular}{|c|c|c|c|c|}
\hline $\begin{array}{l}\text { Histopathological stage } \\
n\end{array}$ & Patch & Plaque & Tumor & $P$ value \\
\hline$n$ & 101 & 50 & & \\
\hline \multicolumn{5}{|c|}{ T cells surface antigens } \\
\hline $\mathrm{CD}^{+}$ & & & & $0.000 \uparrow$ \\
\hline Mean \pm SD & $71.26 \pm 7.72$ & $75.16 \pm 9.12$ & $88.90 \pm 7.68$ & \\
\hline Median; min.--max. & $71.50 ; 51.30-89.10$ & $76.30 ; 48.70-92.20$ & $88.80 ; 75.40-98.70$ & \\
\hline $\mathrm{CD} 4^{+}$ & & & & $0.000 \uparrow$ \\
\hline Mean \pm SD & $44.56 \pm 8.32$ & $47.22 \pm 12.03$ & $77.76 \pm 15.58$ & \\
\hline Median; min.-max. & $44.50 ; 28.30-73.60$ & $47.60 ; 20.60-66.20$ & $79.60 ; 55.50-97.50$ & \\
\hline $\mathrm{CD}^{+}$ & & & & $0.016 \downarrow$ \\
\hline Mean \pm SD & $24.24 \pm 9.32$ & $25.99 \pm 10.52$ & $7.98 \pm 8.56$ & \\
\hline Median; min.-max. & $22.25 ; 9.40-73.30$ & $24.75 ; 11.40-66.30$ & $3.80 ; 0.50-22.50$ & \\
\hline $\mathrm{CD} 4^{+} / \mathrm{CD}^{+}$ & & & & $0.005 \uparrow$ \\
\hline Mean \pm SD & $2.18 \pm 1.16$ & $2.89 \pm 5.23$ & $43.41 \pm 55.33$ & \\
\hline Median; min.-max. & $1.90 ; 0.20-7.80$ & $1.80 ; 0.30-35.30$ & $19.20 ; 2.50-183.20$ & \\
\hline $\operatorname{TCR} \alpha \beta^{+}$ & & & & $0.000 \uparrow$ \\
\hline Mean \pm SD & $66.63 \pm 8.14$ & $69.26 \pm 8.22$ & $86.39 \pm 8.43$ & \\
\hline Median; min.-max. & $67.70 ; 46.10-87.10$ & $69.25 ; 45.80-86.50$ & $85.10 ; 73.80-98.0$ & \\
\hline $\mathrm{TCR} \gamma \delta^{+}$ & & & & $0.006 \downarrow$ \\
\hline Mean \pm SD & $4.35 \pm 2.97$ & $4.26 \pm 2.75$ & $1.28 \pm 4.43$ & \\
\hline Median; min.-max. & $3.60 ; 0.60-13.60$ & $3.35 ; 0.50-11.20$ & $1.20 ; 0.10-10.0$ & \\
\hline $\mathrm{CD}^{+}$ & & & & $0.044 \downarrow$ \\
\hline Mean \pm SD & $76.68 \pm 10.28$ & $71.99 \pm 9.32$ & $73.05 \pm 27.14$ & \\
\hline Median; min.-max. & $78.15 ; 2.80-93.10$ & $72.20 ; 44.50-87.0$ & $73.60 ; 11.70-98.0$ & \\
\hline $\mathrm{CD}^{+} \mathrm{CD}^{+}$ & & & & $0.003 \uparrow$ \\
\hline Mean \pm SD & $38.91 \pm 8.48$ & $35.89 \pm 8.22$ & $51.34 \pm 27.20$ & \\
\hline Median; min.-max. & $9.65 ; 13.40-54.50$ & $35.3 ; 16.0-53.10$ & $48.70 ; 9.60-96.50$ & \\
\hline $\mathrm{CD}^{+} \mathrm{CD}^{-}$ & & & & $0.000 \uparrow$ \\
\hline Mean \pm SD & $7.10 \pm 6.76$ & $10.17 \pm 7.94$ & $27.70 \pm 28.06$ & \\
\hline Median; min.--max. & $5.0 ; 1.0-51.30$ & $7.40 ; 2.20-35.90$ & $20.80 ; 0.50-88.90$ & \\
\hline $\mathrm{CD}_{71}^{+}$ & & & & $0.000 \uparrow$ \\
\hline Mean \pm SD & $4.47 \pm 7.77$ & $3.94 \pm 4.66$ & $22.21 \pm 21.28$ & \\
\hline Median; min.-max. & $2.60 ; 0.10-71.0$ & $2.40 ; 0.30-23.1$ & $13.20 ; 3.60-57.70$ & \\
\hline \multicolumn{5}{|c|}{ B cells surface antigens } \\
\hline $\mathrm{HLA}^{-\mathrm{DR}^{+}}$ & & & & $0.000 \downarrow$ \\
\hline Mean \pm SD & $31.10 \pm 9.76$ & $32.49 \pm 7.39$ & $17.69 \pm 12.73$ & \\
\hline Median; min.--max. & $28.45 ; 11.40-60.20$ & $31.80 ; 18.80-50.40$ & $16.30 ; 2.20-43.70$ & \\
\hline $\mathrm{CD}_{1} 9^{+}$ & & & & $0.020 \downarrow$ \\
\hline Mean \pm SD & $11.55 \pm 4.74$ & $12.84 \pm 4.44$ & $8.11 \pm 5.29$ & \\
\hline Median; min.--max. & $11.40 ; 1.50-24.80$ & $12.75 ; 4.70-22.50$ & $10.0 ; 15.70$ & \\
\hline HLA-DR ${ }^{+} \mathrm{CD}_{1} 9^{+}$ & & & & $0.041 \downarrow$ \\
\hline Mean \pm SD & $10.79 \pm 4.65$ & $11.87 \pm 4.36$ & $6.80 \pm 5.86$ & \\
\hline Median; min.-max. & $10.65 ; 1.20-24.70$ & $11.95 ; 2.90-20.50$ & $7.80 ; 0.30-15.50$ & \\
\hline \multicolumn{5}{|c|}{ NKT cells surface antigens } \\
\hline $\mathrm{CD}^{+}{ }^{+} \mathrm{CD} 16^{+} \mathrm{CD} 56^{+}$ & & & & $0.000 \downarrow$ \\
\hline Mean \pm SD & $3.38 \pm 3.46$ & $3.61 \pm 3.01$ & $1.60 \pm 1.86$ & \\
\hline Median; min.-max. & $2.10 ; 0.20-20.50$ & $2.55 ; 0.50-12.30$ & $0.90 ; 0.20-6.20$ & \\
\hline \multicolumn{5}{|c|}{ NK cells surface antigens } \\
\hline $\mathrm{CD}^{-}{ }^{-} \mathrm{CD} 16^{+} \mathrm{CD} 56^{+}$ & & & & $0.000 \downarrow$ \\
\hline Mean \pm SD & $14.82 \pm 8.14$ & $11.73 \pm 6.30$ & $2.20 \pm 2.35$ & \\
\hline Median; min.-max. & $14.40 ; 2.60-39.80$ & $11.50 ; 2.10-35.80$ & $1.50 ; 0.10-8.0$ & \\
\hline
\end{tabular}

$\downarrow$ : decreasing with stage; $\uparrow$ : increasing with stage. 
TABLE 5: The mean values of all cell surface antigens according to clinical stage.

\begin{tabular}{|c|c|c|c|c|c|}
\hline Clinical $(\mathrm{T})$ stage & $\mathrm{T}_{1}$ & $\mathrm{~T}_{2}$ & $\mathrm{~T}_{3}$ & $\mathrm{~T}_{4}$ & $P$ value \\
\hline$n$ & 47 & 95 & 6 & 16 & \\
\hline \multicolumn{6}{|c|}{ T cells surface antigens } \\
\hline $\mathrm{CD}^{+}$ & & & & & $0.000 \uparrow$ \\
\hline Mean \pm SD & $73.93 \pm 7.07$ & $71.31 \pm 9.01$ & $88.41 \pm 8.67$ & $77.25 \pm 17.64$ & \\
\hline Median; min.-max. & $74.50 ; 62.30-92.60$ & $71.80 ; 48.70-96.50$ & 87.70; 79.10-98.70 & $85.35 ; 43.60-93.20$ & \\
\hline $\mathrm{CD} 4^{+}$ & & & & & $0.000 \uparrow$ \\
\hline Mean \pm SD & $44.63 \pm 9.42$ & $44.49 \pm 8.60$ & $75.18 \pm 19.31$ & $60.27 \pm 24.06$ & \\
\hline Median; min.-max. & $44.70 ; 15.30-66.20$ & $44.70 ; 20.60-63.90$ & $74.35 ; 55.50-97.50$ & $65.20 ; 15.80-92.30$ & \\
\hline $\mathrm{CD}^{+}$ & & & & & $0.005 \downarrow$ \\
\hline Mean \pm SD & $26.75 \pm 10.56$ & $24.12 \pm 9.36$ & $11.41 \pm 10.32$ & $14.23 \pm 9.68$ & \\
\hline Median; min.-max. & $23.20 ; 14.10-73.30$ & $23.45 ; 9.40-66.30$ & $11.60 ; 0.50-22.50$ & $15.10 ; 1.50-32.60$ & \\
\hline $\mathrm{CD} 4^{+} / \mathrm{CD} 8^{+}$ & & & & & $0.000 \uparrow$ \\
\hline Mean \pm SD & $2.7 \pm 5.16$ & $2.19 \pm 1.21$ & $51.10 \pm 74.46$ & $13.97 \pm 20.21$ & \\
\hline Median; min.-max. & $1.90 ; 0.20-35.30$ & $1.80 ; 0.30-7.80$ & $10.35 ; 2.50-183.20$ & $3.90 ; 0.60-61.50$ & \\
\hline $\operatorname{TCR} \alpha \beta^{+}$ & & & & & $0.000 \uparrow$ \\
\hline Mean \pm SD & $68.99 \pm 7.42$ & $66.68 \pm 8.28$ & $86.65 \pm 9.48$ & $71.68 \pm 17.65$ & \\
\hline Median; min.-max. & $68.60 ; 50.0-87.10$ & $68.0 ; 45.80-86.0$ & $86.3 ; 76.90-98.0$ & $76.0 ; 42.0-93.70$ & \\
\hline $\operatorname{TCR} \gamma \delta^{+}$ & & & & & $0.011 \downarrow$ \\
\hline Mean \pm SD & $5.19 \pm 3.30$ & $3.95 \pm 2.63$ & $1.66 \pm 0.92$ & $2.35 \pm 2.66$ & \\
\hline Median; min.-max. & $4.40 ; 0.80-13.60$ & $3.25 ; 0.50-11.20$ & $2.10 ; 0.40-2.60$ & $1.45 ; 0.10-10.0$ & \\
\hline $\mathrm{CD}^{+}$ & & & & & $0.001 \downarrow$ \\
\hline Mean \pm SD & $77.89 \pm 6.33$ & $74.19 \pm 11.33$ & $79.63 \pm 15.65$ & $61.73 \pm 21.63$ & \\
\hline Median; min.-max. & $78.20 ; 58.60-93.10$ & $76.10 ; 2.80-89.30$ & $77.75 ; 54.70-98.0$ & $66.50 ; 11.70-83.0$ & \\
\hline $\mathrm{CD}^{+} \mathrm{CD}^{+}$ & & & & & $0.859 \uparrow$ \\
\hline Mean \pm SD & $38.91 \pm 8.26$ & $37.25 \pm 8.41$ & $64.85 \pm 23.52$ & $37.67 \pm 17.19$ & \\
\hline Median; min.-max. & $40.10 ; 13.40-53.10$ & $37.05 ; 16.0-53.60$ & $54.30 ; 42.50-96.50$ & $35.45 ; 9.60-71.30$ & \\
\hline $\mathrm{CD}^{+} \mathrm{CD}^{-}$ & & & & & $0.002 \uparrow$ \\
\hline Mean \pm SD & $7.71 \pm 7.75$ & $7.81 \pm 6.40$ & $11.40 \pm 16.17$ & $24.02 \pm 25.18$ & \\
\hline Median; min.-max. & $5.70 ; 1.70-51.30$ & $5.20 ; 1.0-35.90$ & $4.65 ; 0.50-42.50$ & $15.75 ; 2.30-88.90$ & \\
\hline $\mathrm{CD} 1^{+}$ & & & & & $0.002 \uparrow$ \\
\hline Mean \pm SD & $3.31 \pm 2.78$ & $4.79 \pm 8.39$ & $14.36 \pm 15.71$ & $17.45 \pm 21.59$ & \\
\hline Median; min.-max. & $2.50 ; 0.3-11.30$ & $3.0 ; 0.10-71.0$ & $7.80 ; 3.60-44.60$ & $6.50 ; 1.4-57.70$ & \\
\hline \multicolumn{6}{|c|}{ B cells surface antigens } \\
\hline $\mathrm{HLA} \mathrm{DR}^{+}$ & & & & & $0.001 \downarrow$ \\
\hline Mean \pm SD & $29.46 \pm 8.79$ & $32.37 \pm 9.11$ & $21.56 \pm 15.76$ & $28.15 \pm 15.30$ & \\
\hline Median; min.-max. & $27.50 ; 19.60-56.40$ & $31.10 ; 11.40-60.20$ & $22.0 ; 3.80-43.70$ & $25.60 ; 2.20-59.90$ & \\
\hline $\mathrm{CD}_{1} 9^{+}$ & & & & & $0.021 \downarrow$ \\
\hline Mean \pm SD & $11.37 \pm 3.96$ & $12.48 \pm 4.89$ & $8.13 \pm 6.41$ & $8.67 \pm 4.37$ & \\
\hline Median; min.-max. & $11.70 ; 1.50-19.10$ & $11.95 ; 3.20-24.80$ & $6.80 ; 1.0-15.70$ & $8.50 ; 2.30-18.10$ & \\
\hline 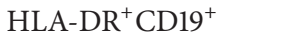 & & & & & $0.030 \downarrow$ \\
\hline Mean \pm SD & $10.53 \pm 3.92$ & $11.64 \pm 4.79$ & $6.78 \pm 7.17$ & $7.72 \pm 4.31$ & \\
\hline Median; min.-max. & $10.60 ; 1.20-17.80$ & $11.20 ; 2.90-24.70$ & $4.75 \pm 0.30-15.50$ & $6.95 ; 0.40-17.40$ & \\
\hline \multicolumn{6}{|c|}{ NKT cells surface antigens } \\
\hline $\mathrm{CD}^{+}{ }^{+} \mathrm{CD} 16^{+} \mathrm{CD} 56^{+}$ & & & & & $0.006 \downarrow$ \\
\hline Mean \pm SD & $3.46 \pm 3.10$ & $3.57 \pm 3.50$ & $2.06 \pm 2.12$ & $1.50 \pm 1.16$ & \\
\hline Median; min.-max. & $2.50 ; 0.30-16.60$ & $2.25 ; 0.20-20.50$ & $1.60 ; 0.20-6.20$ & $1.25 ; 0.20-3.80$ & \\
\hline \multicolumn{6}{|c|}{ NK cells surface antigens } \\
\hline $\mathrm{CD}^{-}{ }^{-} \mathrm{CD} 16^{+} \mathrm{CD} 56^{+}$ & & & & & $0.000 \downarrow$ \\
\hline Mean \pm SD & $13.28 \pm 6.34$ & $14.34 \pm 8.30$ & $2.90 \pm 2.92$ & $8.86 \pm 9.84$ & \\
\hline Median; min.-max. & $13.10 ; 2.60$ & $13.60 ; 2.10-39.80$ & $2.70 ; 0.10-8.0$ & $3.30 ; 0.10-30.20$ & \\
\hline
\end{tabular}

$\downarrow$ : decreasing with stage; $\uparrow$ : increasing with stage. 
TABLE 6: ROC curve analysis of the investigated cell surface antigens to predict the cut-off value.

\begin{tabular}{|c|c|c|c|c|c|}
\hline Cell surface antigen & Cut-off value criterion & Area under curve & $P$ value & Sensitivity (95\% CI) & Specificity $(95 \% \mathrm{CI})$ \\
\hline \multicolumn{6}{|c|}{ T cells surface antigens } \\
\hline $\mathrm{CD}^{+}$ & $>79.1$ & 0.859 & 0.001 & $87.50(61.6-98.1)$ & $84.56(22.5-55.2)$ \\
\hline $\mathrm{CD} 4^{+}$ & $>49.2$ & 0.880 & 0.001 & $87.50(61.6-98.1)$ & $84.56(77.7-90.0)$ \\
\hline $\mathrm{CD}^{+}$ & $\leq 5.2$ & 0.835 & 0.001 & $62.5(35.5-84.7)$ & 98.66 (95.2-99.8) \\
\hline $\mathrm{CD} 4^{+} / \mathrm{CD}^{+}$ & $>2.4$ & 0.873 & 0.001 & $93.75(69.7-99.0)$ & $69.80(61.70-77.0)$ \\
\hline $\operatorname{TCR} \alpha \beta^{+}$ & $>73.1$ & 0.887 & 0.001 & $92.86(66.1-98.8)$ & $72.29(71.6-85.7)$ \\
\hline $\operatorname{TCR} \gamma \delta^{+}$ & $\leq 2.4$ & 0.847 & 0.001 & $85.71(57.2-97.8)$ & $73.38(65.2-80.5)$ \\
\hline $\mathrm{CD}^{+}$ & $\leq 48$ & 0.647 & 0.036 & $78.57(49.2-95.1)$ & $50.74(42.0-59.4)$ \\
\hline $\mathrm{CD}^{+} \mathrm{CD}^{+}$ & $>47.8$ & 0.680 & 0.028 & $50(23.1-76.9)$ & $89.51(83.3-94.0)$ \\
\hline $\mathrm{CD}^{+} \mathrm{CD}^{-}$ & $>9.4$ & 0.660 & 0.053 & $64.29(35.2-87.1)$ & $82.27(74.9-88.2)$ \\
\hline $\mathrm{CD}_{71}^{+}$ & $>3.4$ & 0.831 & 0.001 & $92.86(66.1-98.8)$ & $63.43(54.7-71.6)$ \\
\hline \multicolumn{6}{|c|}{ B cells surface antigens } \\
\hline HLA-DR ${ }^{+}$ & $\leq 23.1$ & 0.734 & 0.001 & $56.25(26.9-80.2)$ & $97.3(93.2-99.2)$ \\
\hline $\mathrm{CD}_{19}{ }^{+}$ & $\leq 6$ & 0.691 & 0.001 & $43.75(19.8-70.1)$ & $89.86(83.8-94.2)$ \\
\hline 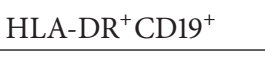 & $\leq 5$ & 0.723 & 0.001 & $56.25(29.9-80.2)$ & $85.14(78.4-90.4)$ \\
\hline \multicolumn{6}{|c|}{ NKT cells surface antigens } \\
\hline $\mathrm{CD}^{+} \mathrm{CD} 16^{+} \mathrm{CD} 56^{+}$ & $\leq 1$ & 0.649 & 0.023 & $68.75(41.4-88.9)$ & $62.16(53.8-70.0)$ \\
\hline \multicolumn{6}{|c|}{ NK cells surface antigens } \\
\hline $\mathrm{CD}^{-} \mathrm{CD}^{-} 6^{+} \mathrm{CD} 56^{+}$ & $\leq 9.9$ & 0.869 & 0.001 & $75.0(47.6-92.6)$ & $92.52(87.0-96.2)$ \\
\hline
\end{tabular}

expression of CD7 and CD26 can be found in patients with benign inflammatory dermatoses and aberrant dim CD3 can be observed in nonneoplastic $\mathrm{T}$ cells [4]. Flow cytometric studies of the peripheral blood may show expansion of the $\mathrm{CD} 4^{+} \mathrm{CD}^{-}$population reflective of circulating atypical lymphocytes of Sezary type [18]. Lymphoma cells in the peripheral blood, especially at high levels, have been recognized as an independent adverse prognostic indicator in patients with mycosis fungoides, and the International Society for Cutaneous Lymphomas and the Cutaneous Lymphoma Task Force of European Organization for Research and Treatment of Cancer revised the mycosis fungoides/Sezary syndrome staging criteria in 2007 to incorporate blood (B) involvement as a major prognostic factor and defined the criteria for B-staging. Natural Killer activity of peripheral blood mononuclear cells in patients with MF was investigated with controlled study and revealed that patients with advanced disease had a significant defect of Natural Killer activity [19]. T cell lymphomas with aberrant CD20 (B cell surface antigen) expression, associated with worse prognosis, have been reported [20-22]. Hristov et al. reported that changes in abnormal populations parallel changes in patient's clinical course and increasing numbers of abnormal $\mathrm{T}$ cells were associated with worsening disease, and authors suggested that flow cytometry provides valuable information for diagnosis, prognosis, and therapeutic efficacy in MF/SS [23].

To the best of our knowledge this is the largest study to assess the prognostic role and clinical utility of flow cytometry in patients with MF. Peripheral blood samples as a diagnostic and tool for MF. Although, in cases of plaque or tumoral stage of MF, immunophenotypical abnormalities are significant, the significance of flow cytometric analysis in early stage MF is limited. Our findings suggest that T cell phenotype affects the clinical behaviour of MF compatible with the previous reports.

\section{Conclusion}

We concluded that flow cytometric analysis of peripheral blood cell surface antigens in patients with mycosis fungoides may contribute to identifying an advanced stage and predicting the disease progression. Flow cytometry is less time consuming and is prognostic tool for the management of MF.

\section{Conflict of Interests}

The authors declare that there is no conflict of interests regarding the publication of this paper.

\section{References}

[1] E. Olsen, E. Vonderheid, N. Pimpinelli et al., "Revisions to the staging and classification of mycosis fungoides and Sezary syndrome: a proposal of the International Society for Cutaneous Lymphomas (ISCL) and the cutaneous lymphoma task force of the European Organization of Research and Treatment of Cancer (EORTC)," Blood, vol. 110, no. 6, pp. 1713-1722, 2007.

[2] H. J. Meyerson, "Flow cytometry for the diagnosis of mycosis fungoides," Giornale Italiano di Dermatologia e Venereologia, vol. 143, no. 1, pp. 21-41, 2008.

[3] M. Lima, J. Almeida, M. dos Anjos Teixeira et al., "Utility of flow cytometry immunophenotyping and DNA ploidy studies for diagnosis and characterization of blood involvement in CD4+ Sezary's syndrome," Haematologica, vol. 88 , no. 8, pp. 874-887, 2003. 
[4] S. Oshtory, N. Apisarnthanarax, A. C. Gilliam, K. D. Cooper, and H. J. Meyerson, "Usefulness of flow cytometry in the diagnosis of mycosis fungoides," Journal of the American Academy of Dermatology, vol. 57, no. 3, pp. 454-462, 2007.

[5] E. Olsen, E. Vonderheid, N. Pimpinelli et al., "ISCL/EORTC. Revisions to the staging and classification of mycosis fungoides and Sezary syndrome: a proposal of the International Society for Cutaneous Lymphomas (ISCL) and the cutaneous lymphoma task force of the European Organization of Research and Treatment of Cancer (EORTC)," Blood, vol. 110, no. 6, pp. 17131722, 2007.

[6] M.-L. Jullié, M. Carlotti, A. Vivot Jr. et al., "CD20 antigen may be expressed by reactive or lymphomatous cells of transformed mycosis fungoides: diagnostic and prognostic impact," American Journal of Surgical Pathology, vol. 37, no. 12, pp. 1845-1854, 2013.

[7] A. E. S. Brooks, "Skin-resident CD4+ T cells express NK receptors: lessons from skin pathologies," Cytometry Part A, vol. 85, pp. 827-829, 2014.

[8] E. A. Olsen, S. Whittaker, Y. H. Kim et al., "Clinical end points and response criteria in mycosis fungoides and Sézary syndrome: a consensus statement of the International Society for Cutaneous Lymphomas, the United States Cutaneous Lymphoma Consortium, and the Cutaneous Lymphoma Task Force of the European Organisation for Research and Treatment of Cancer," Journal of Clinical Oncology, vol. 29, no. 18, pp. 25982607, 2011.

[9] R. T. Hoppe, G. S. Wood, and E. A. Abel, "Mycosis fungoides and the Sézary syndrome: pathology, staging, and treatment," Current Problems in Cancer, vol. 14, no. 6, pp. 295-371, 1990.

[10] J. K. C. Chan, C. S. Ng, and P. K. Hui, "A simple guide to the terminology and application of leucocyte monoclonal antibodies," Histopathology, vol. 12, no. 5, pp. 461-480, 1988.

[11] H. Zola, B. Swart, A. Banham et al., "CD molecules 2006human cell differentiation molecules," Journal of Immunological Methods, vol. 319, no. 1-2, pp. 1-5, 2007.

[12] C. H. Jokinen, J. R. Fromm, Z. B. Argenyi, J. Olerud, B. L. Wood, and H. A. Greisman, "Flow cytometric evaluation of skin biopsies for mycosis fungoides," American Journal of Dermatopathology, vol. 33, no. 5, pp. 483-491, 2011.

[13] K. Kelemen, C. R. White, K. Gatter, R. M. Braziel, and G. Fan, "Immunophenotypic correlation between skin biopsy and peripheral blood findings in mycosis fungoides," American Journal of Clinical Pathology, vol. 134, no. 5, pp. 739-748, 2010.

[14] S. Hara, T. Yokote, T. Akioka et al., "Flow cytometric immunophenotyping of peripheral T cell neoplasms Using CD3 gating," Acta Haematologica, vol. 121, no. 1, pp. 11-18, 2009.

[15] B. Feng, J. L. Jorgensen, D. Jones et al., "Flow cytometric detection of peripheral blood involvement by mycosis fungoides and Sézary syndrome using T-cell receptor Vbeta chain antibodies and its application in blood staging," Modern Pathology, vol. 23, no. 2, pp. 284-295, 2010.

[16] R. M. de Tute, "Flow cytometry and its use in the diagnosis and management of mature lymphoid malignancies," Histopathology, vol. 58, no. 1, pp. 90-105, 2011.

[17] L. C. Edsall and J. W. Patterson, "Cutaneous T-cell lymphoma: important diagnostic tools," Giornale Italiano di Dermatologia e Venereologia, vol. 145, no. 3, pp. 323-344, 2010.

[18] H. Wu, M. Smith, M. M. Millenson et al., "Contribution of flow cytometry in the diagnosis of cutaneous lymphoid lesions," Journal of Investigative Dermatology, vol. 121, no. 6, pp. 15221530, 2003.
[19] S. Levy, J.-L. Tempé, P. Caussade et al., "Stage-related decrease in natural killer cell activity in untreated patients with mycosis fungoides," Cancer Immunology Immunotherapy, vol. 18, no. 2, pp. 138-140, 1984.

[20] N. N. Balmer, L. Hughey, K. J. Busam, V. Reddy, and A. A. Andea, "Primary cutaneous peripheral T-cell lymphoma with aberrant coexpression of CD20: case report and review of the literature," The American Journal of Dermatopathology, vol. 31, no. 2, pp. 187-192, 2009.

[21] A. Garcia-Herrera, L. Colomo, M. Camós et al., "Primary cutaneous small/medium CD4+ T-cell lymphomas: a heterogeneous group of tumors with different clinicopathologic features and outcome," Journal of Clinical Oncology, vol. 26, no. 20, pp. 3364-3371, 2008.

[22] G. Ferrara, C. Chiarelli, and S. Simonetti, "B-cell lymphofollicular infiltrates in mycosis fungoides," Tumori, vol. 96, no. 3, pp. 487-491, 2010.

[23] A. C. Hristov, E. C. Vonderheid, and M. J. Borowitz, "Simplified flow cytometric assessment in mycosis fungoides and sézary syndrome," American Journal of Clinical Pathology, vol. 136, no. 6, pp. 944-953, 2011. 


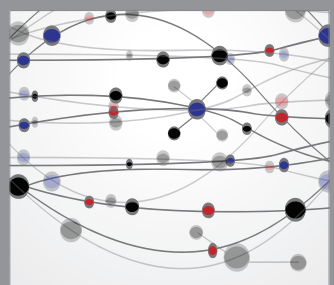

The Scientific World Journal
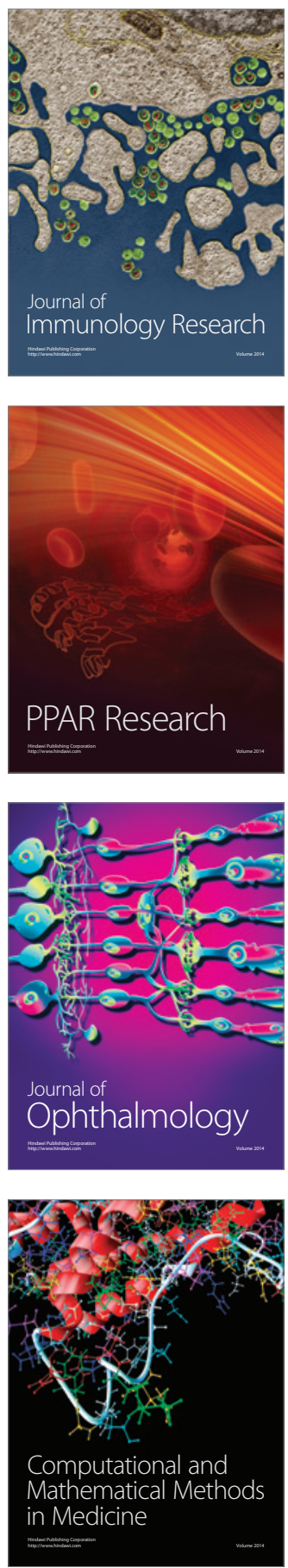

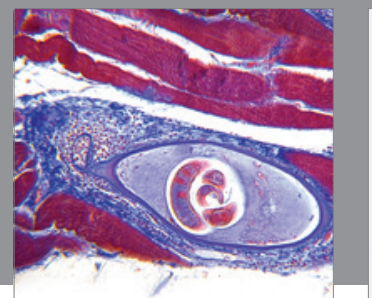

Gastroenterology

Research and Practice
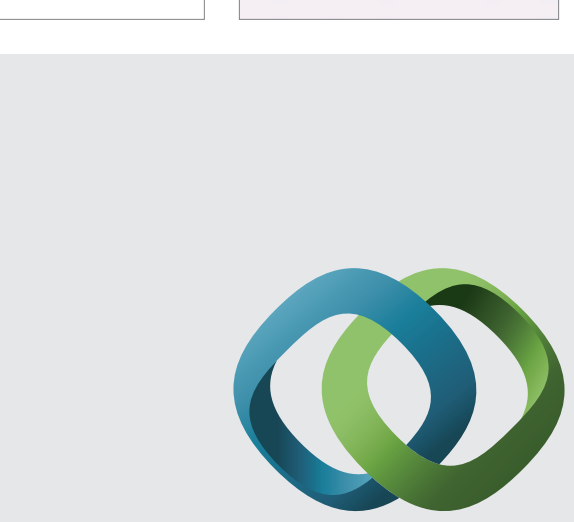

\section{Hindawi}

Submit your manuscripts at

http://www.hindawi.com
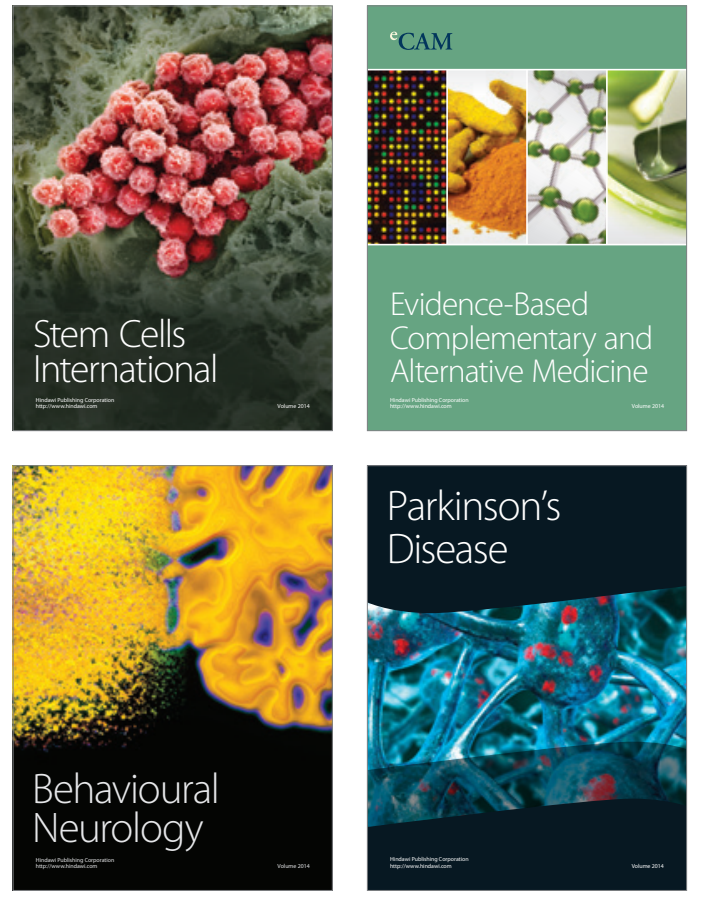
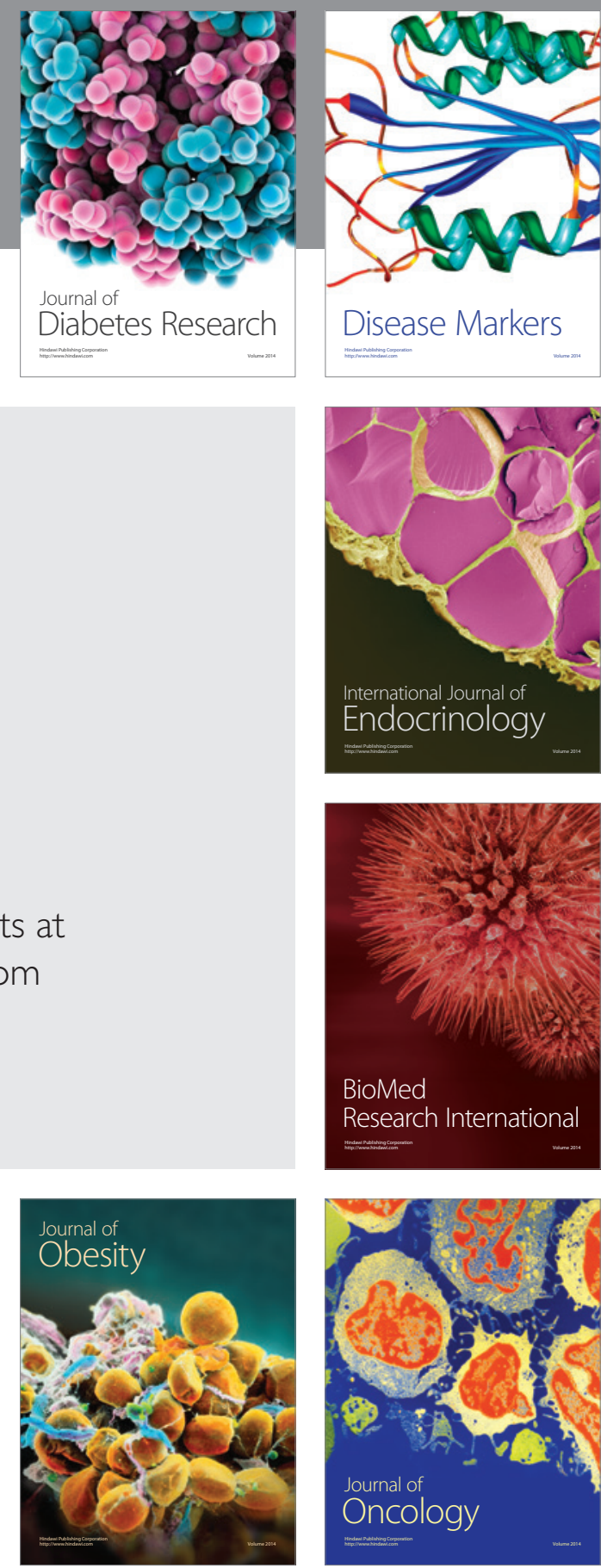

Disease Markers
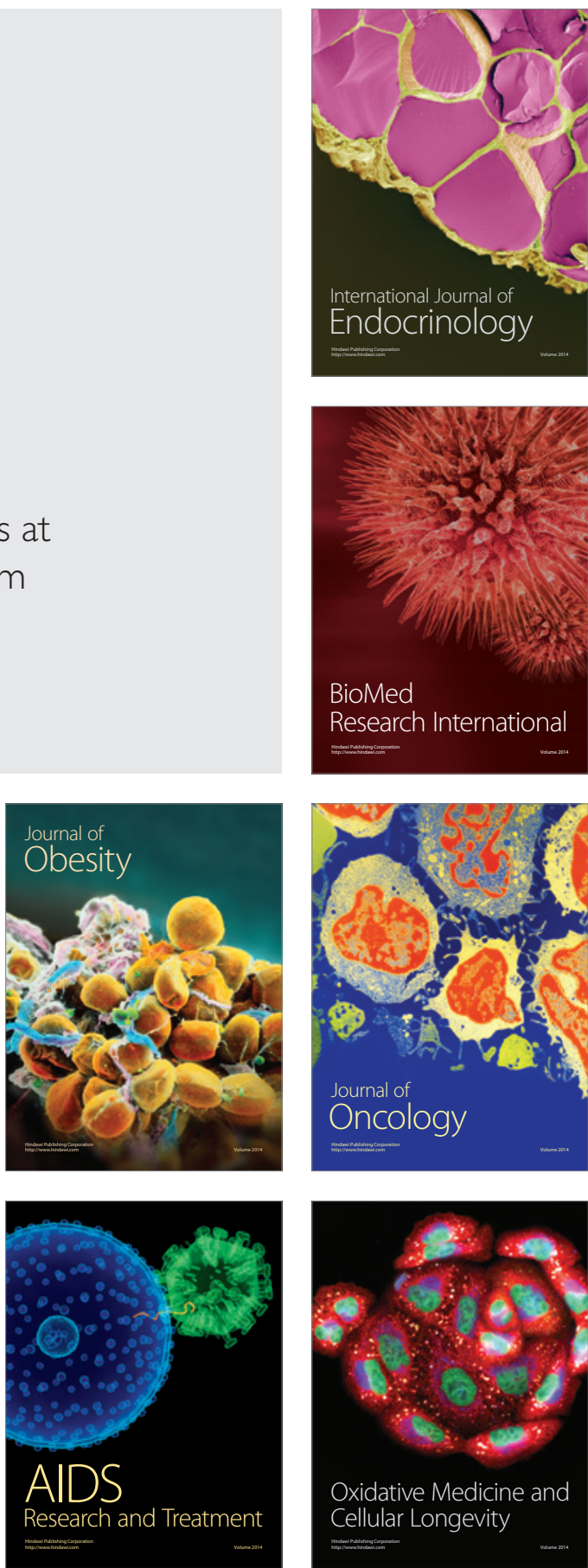